\title{
NOISE AND VIBRATION EXPOSURES TO DENTAL HYGIENISTS
}

\author{
Donald R. Peterson*, Takafumi Asaki, Anthony J. Brammer, Martin G. Cherniack \\ Biodynamics Laboratory, Univ. of Conn. Health Ctr., Farmington, CT 06030-2017
}

\section{Introduction}

The association of hand paresthesias and auditory damage to exposures of highfrequency sound and vibration from dental instrumentation remains unclear. Auditory threshold studies have shown dental practitioners to have significant hearing loss at 3 $\mathrm{kHz}, 4 \mathrm{kHz}$, and $6 \mathrm{kHz}{ }^{1,2}$. Experienced hygienists were observed to have elevated Vibration Perception Thresholds (VPTs) at the FAII mechanoreceptors ${ }^{3}$ even though daily vibration exposures are below the limit value of the ISO 5349-based European Union Directive ${ }^{4}$. These studies suggest that years of high-frequency exposures may lead to symptoms of Hand-Arm Vibration Syndrome (HAVS) and hearing loss. This paper explores these exposure-response relationships using data from questionnaires, vibrotactile testing, and laboratory measurements of high-frequency dental instruments.

\section{Methods}

Two populations of dental hygienists (hygiene students $(n=66)$, and experienced hygienists ( $n=94$ ) with at least five years of experience) were recruited to investigate the relationships between multiple exposures and symptoms, as well as the early onset of symptoms in previously unexposed students ${ }^{3}$. Job information was obtained by questionnaire and vibrotactile perception thresholds were measured for the FAII (125 $\mathrm{Hz}$ ), FAI (32 Hz), and SAI (4 Hz) mechanoreceptors on both hands using a tactometer. (Auditory thresholds were not collected.) The most commonly used vibratory dental instruments were identified from the questionnaire data and the weighted and unweighted 1/3 octave band frequency spectra of sound and vibration up to $63 \mathrm{kHz}$ were measured using a 1/4-inch free-field microphone (4939, B\&K, Denmark) coupled with an ultra high-frequency Scanning Laser Vibrometer (PSV-300, Polytec GmbH, Germany). Instruments were operated without load (i.e., no tip contact) at recommended operating pressures and were evaluated using two mounting positions (tool base and typical grip position) using a simulated pinch grip with a similar biodynamic response to a human three-finger grip for grip forces between 30 and $45 \mathrm{~N}^{3}$.

Table 1: Questionnaire Results ${ }^{3}$

\section{Results and Discussion}

\begin{tabular}{l|c|c} 
& $\begin{array}{c}\text { STUDENTS } \\
\text { (n=66, 98.5\% Female) }\end{array}$ & $\begin{array}{c}\text { EXPERIENCED } \\
(\mathbf{n = 9 4 , 9 7 . 9 \%} \text { Female) }\end{array}$ \\
\hline Age (SD) & $26.1(6.4)$ & $45.5(8.8)$ \\
\hline Years in Practice (SD) & $3.0(4.3)$ & $21.8(8.3)$ \\
\hline Vibration Exposure (years (SD)) & $1.0(1.9)$ & $17.1(8.7)$ \\
\hline Manual Tool Use (hours/week (SD)) & $5.2(5.5)$ & $12.0(7.3)$ \\
\hline Vibratory Tool Use (hours/week (SD)) & $3.0(3.9)$ & $5.1(5.4)$ \\
\hline Est. Lifetime Ave. Weekly Vib. Exp. (hrs*1000 (SD)) & $0.3(0.6)$ & $10.8(11.5)$ \\
\hline Presence of Musculoskeletal Pain (number (\%)) & $27(40.9)$ & $81(86.7)$ \\
\hline Presence of Carpal Tunnel Syndrome (number (\%)) & $3(4.6)$ & $17(18.1)$ \\
\hline Use of Hearing Protection (number (\%)) & $2(3.0)$ & $2(2.1)$
\end{tabular}

Questionnaire results (Table 1) showed significant differences between mean age, years in practice, and self-reported vibration exposures. Experienced hygienists were shown to be four times more likely to suffer from musculoskeletal pain and carpal tunnel 
syndrome and only four hygienists (two students and two experienced) reported the use of hearing protection. Average VPTs (Table 2) for the FAII mechanoreceptors of the third and fifth digits of both hands were observed to be slightly higher for the experienced hygienists, while FAI and SAI results showed no differences and there was no association with age for any of the threshold measurements. The sound and vibration levels of the dental instruments varied and the spectra indicated small differences in frequency components between mounting conditions. Table 3 shows the measured unweighted and weighed total band powers for the SPL and the VL. While the weighted results suggest minimal effects on health, the unweighted results, self-reported exposures, and VPTs suggest that accumulated exposures over time may cause musculoskeletal discomfort, HAVS, and possibly hearing loss.

Table 2: Average Vibration Perception Threshold Results in $\mathrm{dB}\left(\mathrm{re} 1 \times 10^{-6} \mathrm{~m} / \mathrm{s}^{2}\right)$

\begin{tabular}{l|c|c|c}
\multicolumn{1}{c}{ HAND AND DIGIT } & MECHNORECEPTOR & STUDENTS & EXPERIENCED \\
\hline Dominant Hand, 3rd Digit & FAII $-125 \mathrm{~Hz}$ & $103.0(7.5)$ & $107.0(8.8)$ \\
(Median Nerve) & FAI $-32 \mathrm{~Hz}$ & $102.7(6.8)$ & $104.4(6.3)$ \\
& SAI $-4 \mathrm{~Hz}$ & $83.4(4.5)$ & $83.6(5.1)$ \\
\hline Dominant Hand, 5th Digit & FAII $-125 \mathrm{~Hz}$ & $100.6(6.2)$ & $104.3(8.8)$ \\
(Ulnar Nerve) & FAI $-32 \mathrm{~Hz}$ & $104.0(5.7)$ & $104.5(11.8)$ \\
& SAI $-4 \mathrm{~Hz}$ & $83.8(3.6)$ & $84.2(4.5)$ \\
\hline Non-Dominant Hand, 3rd Digit & FAII $-125 \mathrm{~Hz}$ & $101.3(7.8)$ & $105.2(9.0)$ \\
(Median Nerve) & FAI $-32 \mathrm{~Hz}$ & $102.5(6.0)$ & $103.8(6.5)$ \\
& SAI $-4 \mathrm{~Hz}$ & $83.3(4.3)$ & $83.2(4.8)$ \\
\hline Non-Dominant Hand, 5th Digit & FAII $-125 \mathrm{~Hz}$ & $98.9(5.5)$ & $103.2(9.3)$ \\
(Ulnar Nerve) & FAI - 32 Hz & $102.9(7.2)$ & $104.2(7.2)$ \\
& SAI $-4 \mathrm{~Hz}$ & $83.4(4.0)$ & $84.6(5.1)$
\end{tabular}

Table 3: Sound Pressure Level (SPL) and Vibration Level (VL) Measurements

\begin{tabular}{|c|c|c|c|c|c|c|c|}
\hline \multirow[t]{2}{*}{ TYPE } & \multirow[t]{2}{*}{ MODEL } & \multirow[t]{2}{*}{ MOUNT } & \multirow{2}{*}{$\begin{array}{c}\text { OP. FREQ. } \\
(\mathrm{kHz})\end{array}$} & \multicolumn{2}{|c|}{ SPL (re $\left.2 \times 10^{-5} \mathrm{~Pa}\right)$} & \multicolumn{2}{|c|}{ VL (re $\left.1 \times 10^{-6} \mathrm{~m} / \mathrm{s}^{2}\right)$} \\
\hline & & & & dB & $\mathrm{dB}(\mathrm{A})$ & dB & dB(Weighted) \\
\hline \multirow{4}{*}{$\begin{array}{l}\text { Rotary } \\
\text { Polisher }\end{array}$} & $5 \mathrm{k}$ & GRIP & 4.4 & 69.2 & 65.9 & 169.2 & 97.6 \\
\hline & $\mathrm{RDH}$ & GRIP & 4.4 & 77.4 & 72.5 & 168.7 & 98.1 \\
\hline & Hygiene & GRIP & 5.0 & 72.7 & 68.9 & 171.8 & 101.0 \\
\hline & Titan & GRIP & 4.6 & 70.2 & 67.7 & 165.9 & 94.9 \\
\hline \multirow{4}{*}{$\begin{array}{l}\text { Sonic } \\
\text { Scaler }\end{array}$} & Quixonic & BASE & 6.5 & 73.8 & 73.0 & 199.0 & 46.4 \\
\hline & & GRIP & 6.5 & 77.6 & 77.2 & 198.7 & 46.1 \\
\hline & Pirouette & BASE & 6.3 & 80.2 & 75.4 & 176.4 & 51.9 \\
\hline & & GRIP & 6.3 & 81.8 & 79.7 & 171.4 & 54.2 \\
\hline \multirow{4}{*}{$\begin{array}{l}\text { Ultrasonic } \\
\text { Scaler }\end{array}$} & Cavitron & BASE & 29.0 & 102.0 & 75.6 & 192.3 & 78.3 \\
\hline & & GRIP & 29.0 & 99.6 & 75.2 & 198.0 & 76.6 \\
\hline & Advantage & BASE & 27.8 & 99.3 & 48.5 & 201.4 & 83.0 \\
\hline & & GRIP & 27.8 & 94.7 & 46.9 & 197.2 & 65.1 \\
\hline
\end{tabular}

\section{References}

1. Taylor W., Pearson J., Mair A. (1965). The hearing threshold levels of dental practitioners exposed to air turbine drill noise. Br. Dental J. 118, 206-210.

2. Wilson J.D., Darby M.L., Tolle S.L., Sever J.C. (2002). Effects of occupational ultrasonic noise exposure on hearing of dental hygienists: A pilot study. J. Dental Hyg. 76 (4), 262-269.

3. Cherniack M., Brammer A.J., Nilsson T., Lundstrom R., Meyer J.D., Morse T., Neely G., Peterson D., et al. (2006). Nerve conduction and sensorineural function in dental hygienists using high frequency ultrasound handpieces. Am. J. Ind. Med. 49, 313-326.

4. Mansfield, N.J. (2005). The European vibration directive-how will it affect the dental profession? Br. Dental J. 199, 575-577. 